\title{
MAGNET WAVE PROPAGATION
}

\author{
AD \\ BOOSTER TECHNICAL NOTE \\ NO. 136
}

M. METH

JANUARY 30, 1989 


\title{
MAGNET WAVE PROPAGATION
}

\author{
M. METH \\ January 30, 1989
}

\section{INTRODUCTION}

The application of a voltage pulse to the AGS Booster magnet string produces an initial transient response that is characterized as a wave. The wave response results from the distributed inductance and capacitance of each magnet around the ring structure and is similar to the response of a short-circuited transmission line. The wiring of the dipole magnet string is shown in Figure 1. The wave propagates within the magnet string and results in magnetic fields that vary with position around the ring structure. The wave is damped by losses associated with the iron laminations, coil winding and by an external resistor shunted across each half coil of the magnet structure. Following the damping of the wave, the magnet string behaves as a lumped-constant inductor and the magnetic field is uniform around the ring structure.

\section{Model of Magnet}

The electrical model of the dipole magnet is highly frequency dependent and consists of a series inductor and resistor and a shunt capacitor. The frequency dependence of the model is due to flux shielding by the iron, with the value of the series inductor varying from that of an iron-core (low frequency value) to an air-core (high frequency value) with an equivalent winding configuration.

The wave response is highly dispersive and is described by an array of space harmonics. The high order harmonics are subject to a larger damping ratio than the low frequency harmonics due to higher losses which are dependent on skin effect and eddy currents.

The approach taken is to establish two fixed-parameters equivalent circuits: a low frequency (or dominant mode) model and a limiting high frequency model. The low frequency model uses measured component values or calculated parameters. The high frequency model uses parameter values, established from reflectometer measurements of a single magnet. The dominant model response is calculated using the former model; the high frequency response, using the latter model.

The equivalent circuit of the magnet is given in Figure 2 with parameter values listed in Table I. The reflectometers measurements are discussed in Appendix A. 
TABLE I

PARAMETER VALUES OF EQUIVALENT CIRCUIT

\begin{tabular}{|l|l|l|}
\hline PARAMETERS & $\begin{array}{c}\text { DOMINANT } \\
\text { MODE }\end{array}$ & $\begin{array}{c}\text { HIGH FREQUENCY } \\
\text { MODE }\end{array}$ \\
\hline Frequency & & $416 \mathrm{Khz}$ \\
L (per section) & $4.9 \mathrm{Khz}$ & $294 \mu \mathrm{H}$ \\
C (per section) & $3.2 \mathrm{mH}$ & $.005 \mu \mathrm{F}$ \\
R (per section) & $.01 \mu \mathrm{F}$ & $.118 \Omega$ \\
Zo (surge impedance) & $12.6 \times 10^{-3} \Omega$ & $245 \Omega$ \\
$\tau$ (delay per sections) & $565 \Omega$ & $1.2 \mu \mathrm{sec}$ \\
\hline
\end{tabular}




\section{Wave Response}

The magnet wave response can be related to the response of a smooth loss-free, shortcircuited transmission line excited by a voltage step. The voltage and current response at the center of this line is given in Figure 3, where

$$
\begin{aligned}
& \mathrm{V}=\text { magnitude of exciting voltage } \\
& \mathrm{Zo}=\sqrt{\frac{\mathrm{L}}{\mathrm{C}}}=\text { surge impedance of line } \\
& \tau=\mathrm{N} \sqrt{\mathrm{LC}}=\text { one way delay of line } \\
& \mathrm{N}=\text { length of line }
\end{aligned}
$$

Note that the average value of current increases with a slope given by

$$
\frac{\mathrm{d} i}{\mathrm{dt}}=\frac{\mathrm{v}}{\mathrm{z}_{\mathrm{O}}} \frac{1}{\tau}=\frac{\mathrm{V}}{\mathrm{NL}}
$$

At a time $t$ following the application of the driving voltage, the ratio of the current wave propagating in the line $(\Delta \mathrm{i})$ to the average current in the line (i) is given by

$$
\frac{\Delta i}{i}=\frac{N \sqrt{L C}}{t}
$$

To reach a steady state or lumped constant response, the propagating wave is damped by the series loss element $\mathrm{R}$. After traveling a distance $\mathrm{d}$ on the line, the propagating wave is damped to the value

$$
\begin{aligned}
& \Delta i=V \sqrt{\frac{C}{L}} e^{-\alpha d} \\
& \text { where } \alpha=\frac{R}{2 \mathrm{ZO}} \text { nepers/length }=\text { attentuation constant of line. }
\end{aligned}
$$

Thus,

$$
\frac{\Delta i}{i}=\frac{N \sqrt{L C}}{t} \quad e^{-\alpha d}
$$

With the velocity of propagation $\nu$ given by

$$
\nu=\frac{1}{\sqrt{\mathrm{LC}}}
$$

and the distance $\mathrm{d}$ traveled by the initial step, as

$$
\begin{aligned}
& \mathrm{d}=\frac{\mathrm{t}}{\sqrt{\mathrm{LC}}} \\
& \text { thus } \alpha \mathrm{d}=\frac{\mathrm{R}}{2 \mathrm{~L}} \mathrm{t}
\end{aligned}
$$


and $\frac{\Delta i}{i}$ is given by

Wave Damping

$$
\frac{N \sqrt{L C}}{t} \cdot e^{-\frac{R}{2 L t}}
$$

As a reasonable criterion for wave damping let $\frac{\Delta i}{i}$ be .01 at a time $t$ of 1 milliseconds. Thus,

$$
\frac{\Lambda i}{i}=50 \times 10^{-6}
$$

at $t$ equal to 2 milliseconds. This specification is based on the 8 millisecond restraint placed on the power supplies to change voltage from full invert to full rectify.

Using the limiting high frequency values from Table $\mathbf{I}$, the value of $\mathbf{R}$ is calculated as

$$
\mathrm{R}=0.453 \Omega
$$

To damp the dominant mode the value of $\mathrm{R}$ is calculated as

$$
\mathrm{R}=14.72 \Omega
$$

These damping calculations involve a series loss element.

The embodiment of the losses is through a shunt resistance $R_{D}$ as is shown in Figure 4. The equivalence requires a shunt resistance of value

$$
\mathrm{R}_{\mathrm{D}}=4 \mathrm{~K} \Omega
$$

for high frequency damping and

$$
\mathrm{R}_{\mathrm{D}}=659 \Omega
$$

for low frequency damping.

Thus by shunting a $330 \mathrm{n}$ resistor across each half coil, the magnet wave is damped in a time of less than 2 milliseconds.

It is interesting to note that $659 \Omega$ is nearly the low frequency characteristic resistance of each section, resulting in a Qo (quality factor) of 1 , and a system that is slightly under damped. ing resistor.

For an iterative structure, the current in the coil is not influenced by the value of the damp- 


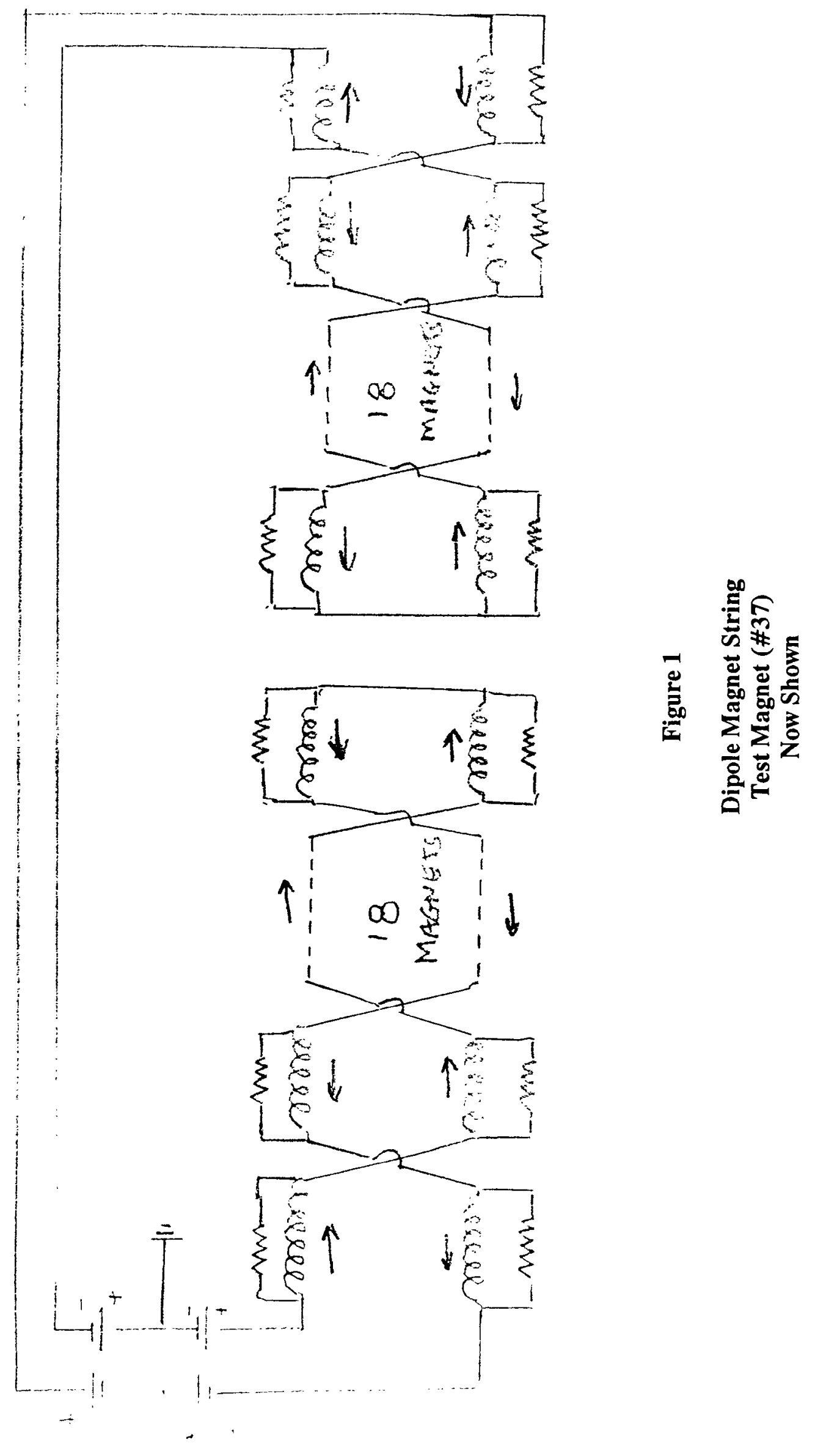




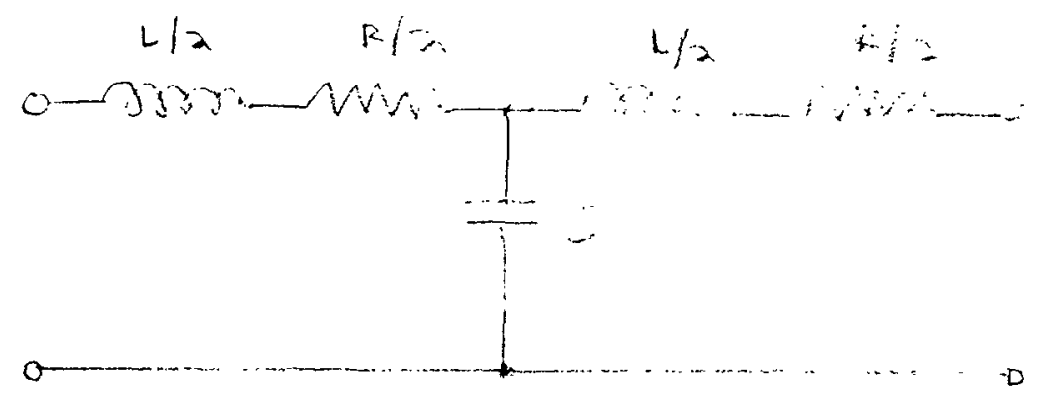

Figure 2

\section{Single Frequency Equivalent Circuit of Dipole Magnet}
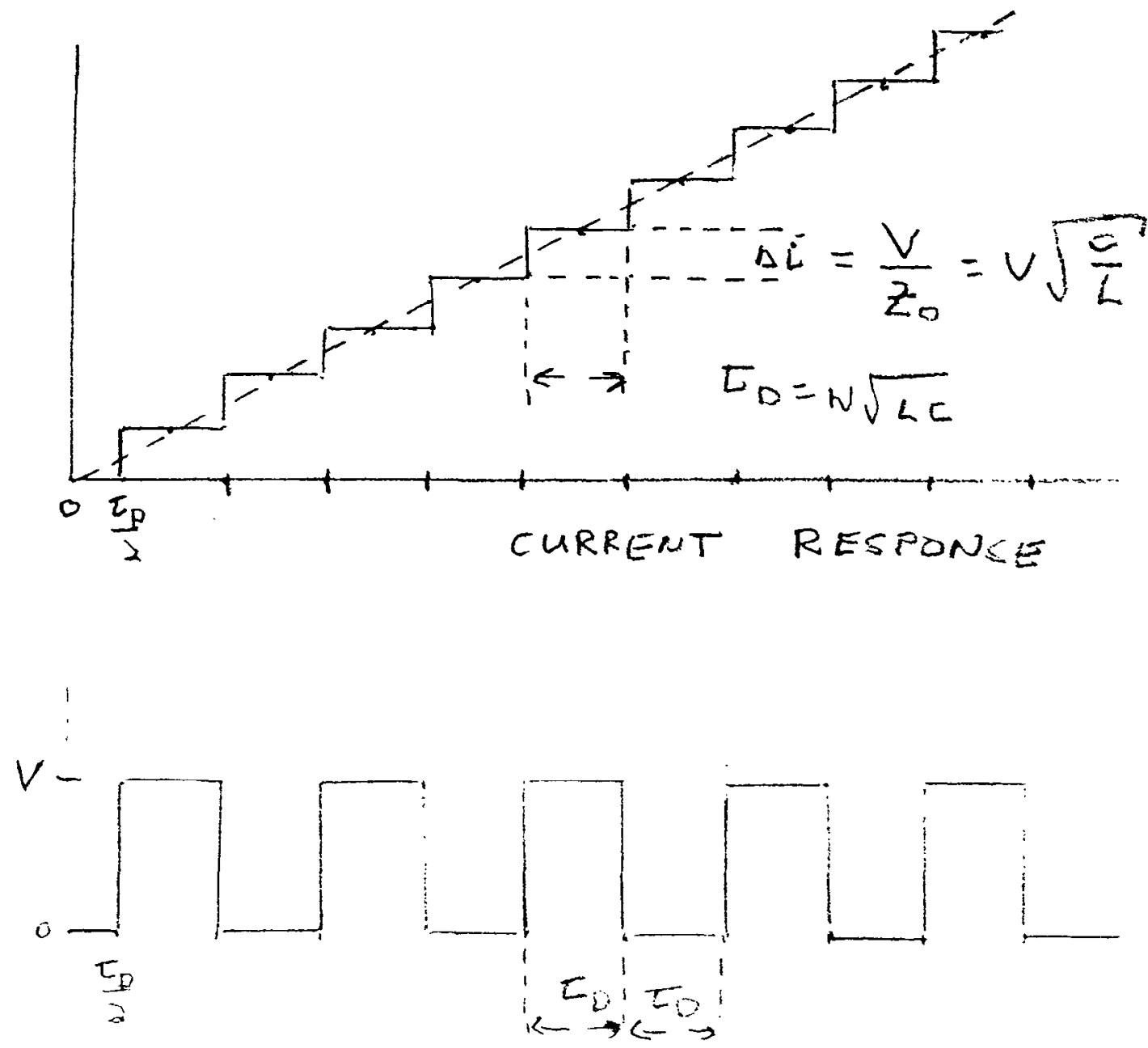

$$
\text { Voltage Response }
$$

Figure 3 


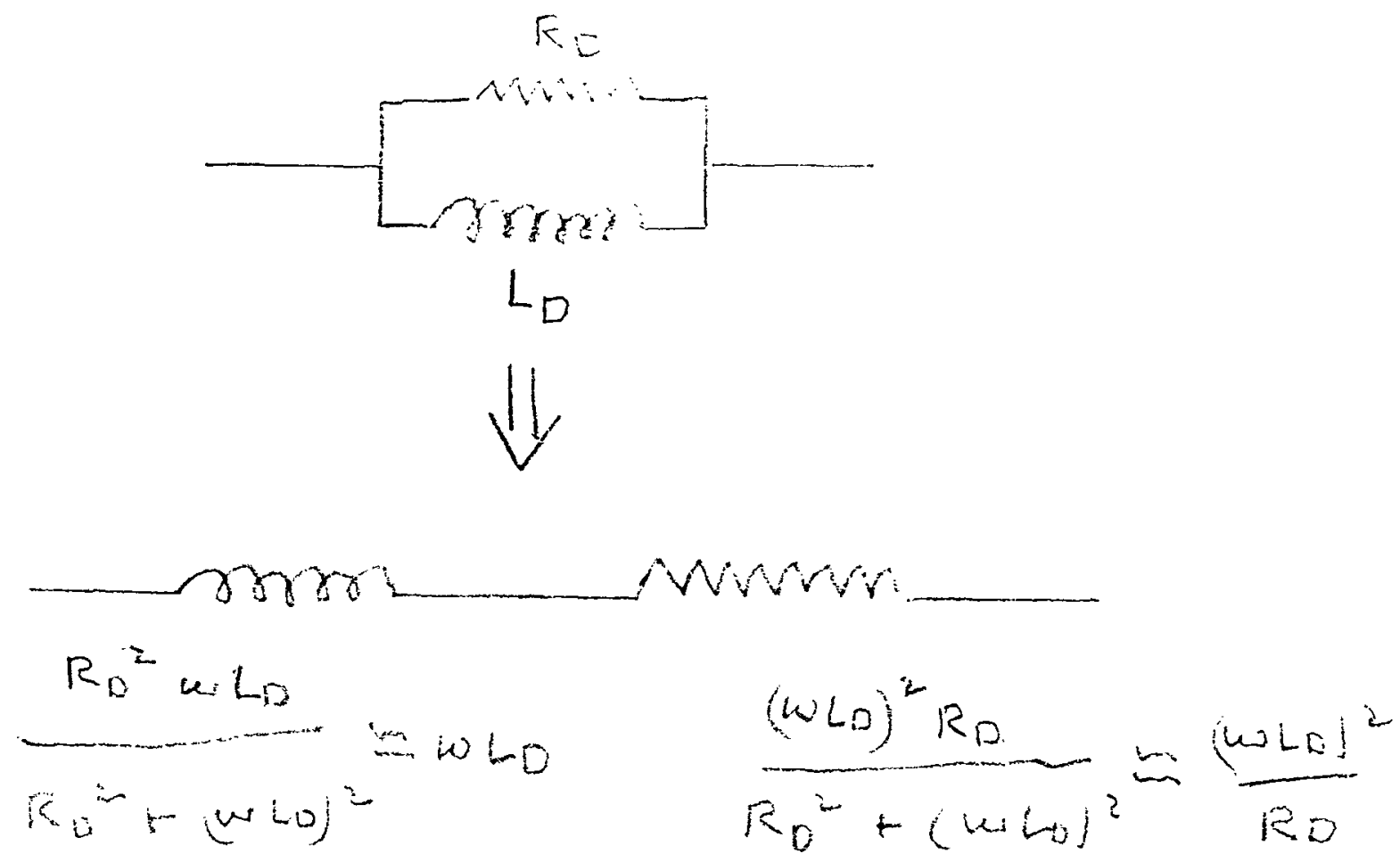

Figure 4

Shunt Loss Element

$$
\mathbf{W L}_{\mathbf{D}}>\mathbf{R}_{\mathbf{D}}
$$

7 


\section{Appendix A}

\section{Reflectometer Measurement of Magnet}

A fast voltage step was applied to the magnet through an adjustable resistor as is shown in Figure A-1.

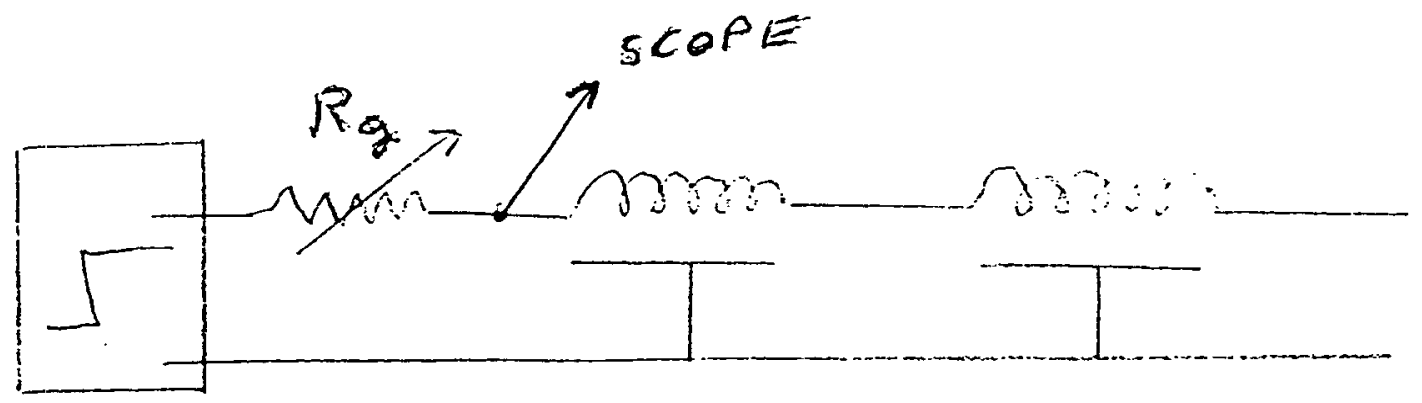

Figure A-1

\section{Reflectometer Test of Dipole Magnet}

The source resistance $\mathrm{R}_{\mathrm{g}}$ was adjusted to eliminate multipole reflections. The oscillogram in Figure A-2 gives the sending end and receiving end voltages for an open circuited line. The oscillogram in Figure A-3 gives the sending end voltage for a short circuited line. For both tests $R_{g}$ was $245 \Omega$. In Figure A-2 note that the initial sending end voltage is $1 / 2$ the final value. In both oscillograms note the time delay of the reflection is approximately $2.4 \mu \mathrm{sec}$. Thus, the values of $\mathrm{Z}_{0}$ and $\tau$ are measured as

$$
\mathrm{Z}_{\mathrm{o}}=245 \Omega
$$

and

$$
\tau=1.2 \mu \mathrm{sec}
$$

and are used to establish Table I. 


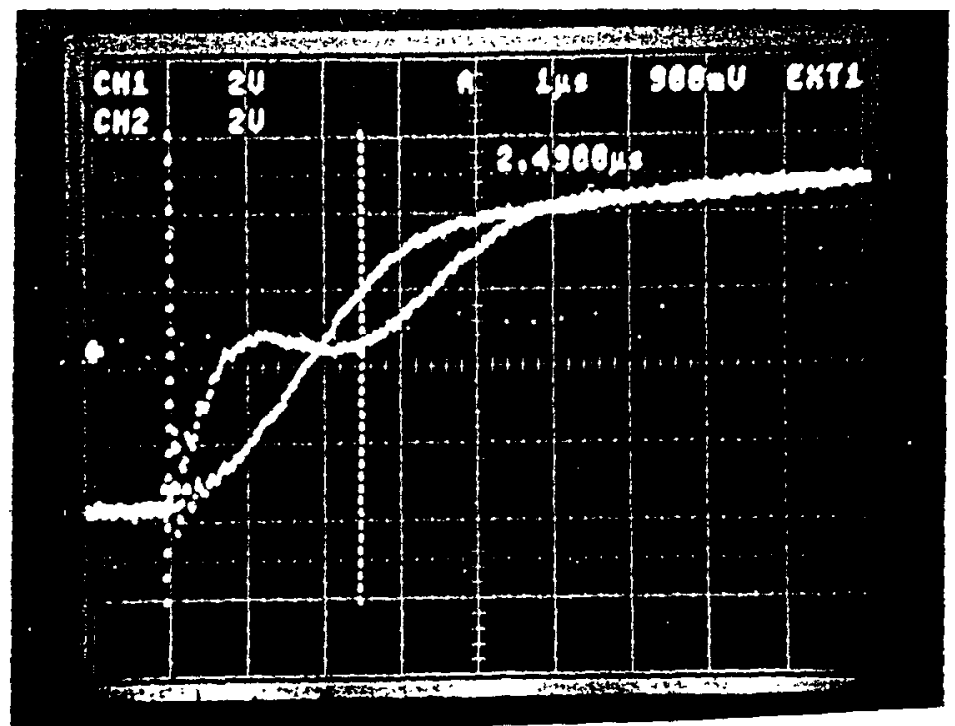

Figure A-2

Response of Open Circuit Line

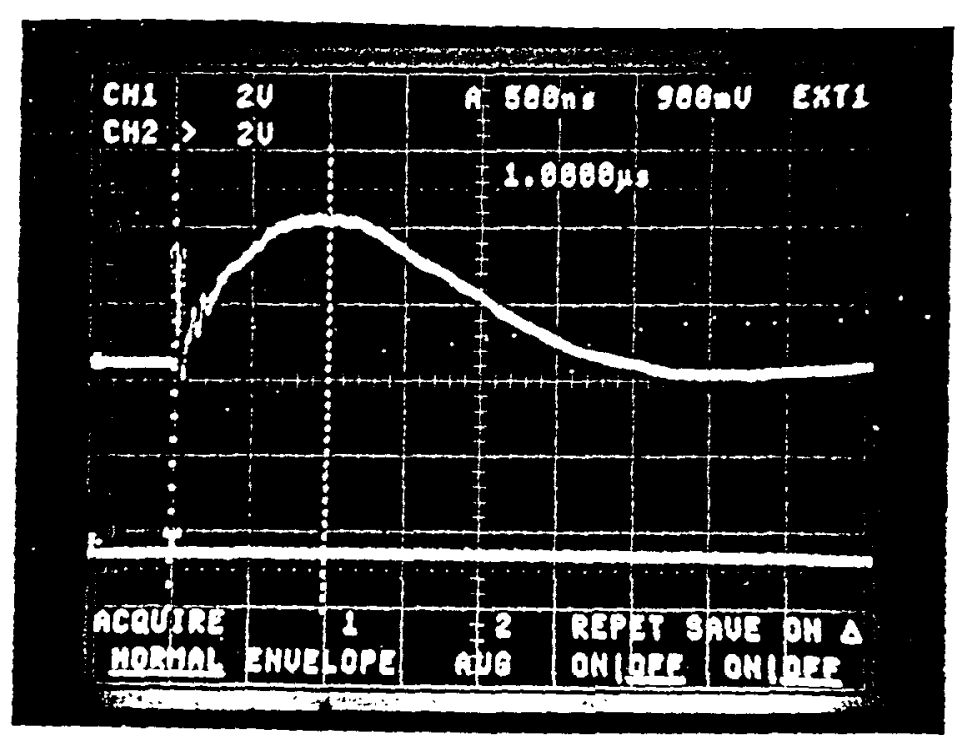

Figure A-3

Response of Short Circuit Line 